\title{
Impact of External Temperature Environment on Large FCBGA Sn-Ag-Cu Solder Interconnect Board Level Mechanical Shock Performance
}

\author{
Tae-Kyu Lee*, $\dagger$ \\ *Component Quality and Technology Group, Cisco Systems, Inc., San Jose, CA 95134 \\ †Corresponding author : taeklee@cisco.com \\ (Received June 24, 2014 ; Accepted June 26, 2014)
}

\begin{abstract}
The mechanical stability of solder joints in electronic devices with $\mathrm{Sn}-\mathrm{Ag}-\mathrm{Cu}$ is a continuous issue since the material was applied to the industry. Various shock test methods were developed and standardized tests are used in the industry worldwide. Although it is applied for several years, the detailed mechanism of the shock induced failure mechanism is still under investigation. In this study, the effect of external temperature was observed on large Flip-chip BGA components. The weight and size of the large package produced a high strain region near the corner of the component and thus show full fracture at around 200G level shock input. The shock performance at elevated temperature, at $100^{\circ} \mathrm{C}$ showed degradation based on board pad designs. The failure mode and potential failure mechanisms are discussed
\end{abstract}

Key Words : Solder, Mechanical shock, FCBGA, Reliability

\section{Introduction}

With more function and capacity on a single Silicon chip, we can see a higher density component trend in one side, like consumer electronics which needs smaller form factor and lighter packages to fit their product design features. But on the other side, the higher functionality and at the same time the high speed capacity brings the component to a larger silicon die, with ending up in a larger than ever body size component. It is not unusual to see a $50+\mathrm{mm}$ package in the telecom industry these days. With larger body size and heavier weight per components, the solder joints have a higher chance to experience a greater challenge for mechanical shock. It is well known that Tin based solder alloy, like $\mathrm{Sn}-\mathrm{Ag}-\mathrm{Cu}$ solder joints are more rigid from a mechanical property standpoint, due to its higher modulus and long stress relaxation time, than conventional $\mathrm{Sn}-\mathrm{Pb}$ eutectic solder joints. Because of this property, exposed to dynamic operating conditions, such as shock and bending, has been a point of concern $^{1-6)}$. Another factor to consider is the temperature of the component in full function. Since these large BGA components are high performance components and their junction temperature easily brings this component to a higher temperature state. Thus, the shock performance needs to be observed not only at room temperature but also at elevated temperatures, which is expected to be different because the mechanical properties of the $\mathrm{Sn}-\mathrm{Ag}-\mathrm{Cu}$ solder joint is expected to be different at higher temperatures. This study presented here, is subject to see the overall shock performance of a $52.5 \times$ $52.5 \mathrm{~mm}^{2}$ Flip Chip ball grid array (FCBGA) component at both room temperature and $100^{\circ} \mathrm{C}$ temperature environment. The failure modes and mechanism will be discussed. 


\section{Experimental Procedure}

In this study observing the shock performance, 1.0-mm-pitch daisy-chained full-array flip chip ball grid array (FCBGA) packages with a size of $52.5 \mathrm{~mm} \times 52.5 \mathrm{~mm}$ were used. Each package was assembled on $2.36 \mathrm{~mm}$ eight-layered print circuit board (PCB) with adequate connection to form two separate daisy chain circuits as shown in Fig. 1(a). Two pad designs were selected to see the performance difference between solder mask defined (SMD) and Nonsolder mask defined (NSMD) pad designs. The illustrations for both pad designs are shown in Fig. 1(b). Regarding the monitoring and connectivity measurement during shock, one daisy chain is connected to the corner solder joints to monitor the solder joint connection during shock test and the other daisy chain was distributed under the silicon die area to provide as a heat source during the test. A calibrated current was passed through the inner daisy chain region to bring the temperature up to $100^{\circ} \mathrm{C}$ at the corner joints, which was measured by a K-type thermocouple. $\mathrm{Sn}-3.0 \mathrm{Ag}-0.5 \mathrm{Cu} \quad(\mathrm{wt} \%)$ (SAC305) solder alloy solder joints and solder paste were used for assembly. The PCB used was an organic solderability preservative (OSP) finish high-Tg FR4 test board. As seen in Fig. 1(a), the mounting holes were located in a $127 \mathrm{~mm} \times 127 \mathrm{~mm}$ con- $^{-}$
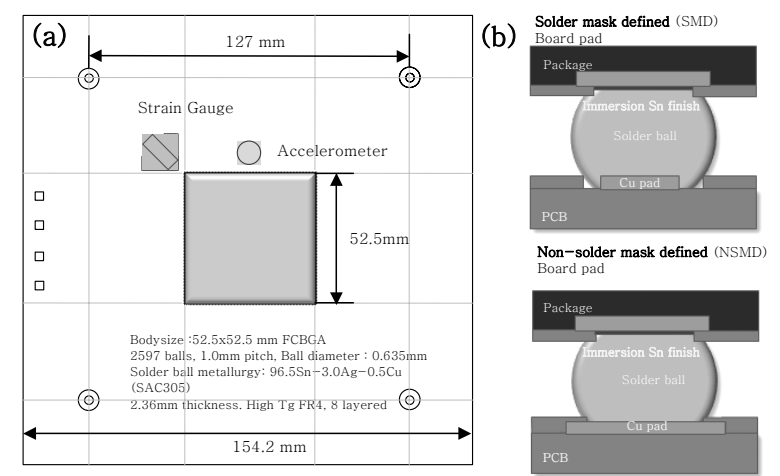

Fig. 1 (a) Shock test board configuration (b) schematically shown board side solder mask defined (SMD) and Non-solder mask defined (NSMD) pad design

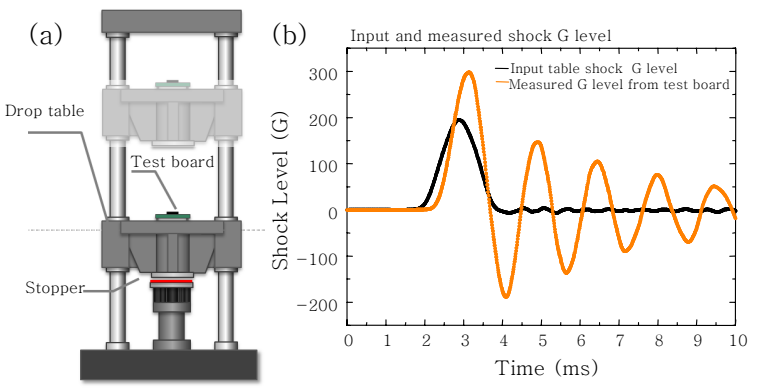

Fig. 2 (a) Shock test set up and (b) measured input shock level and $G$ level at the component edge on the board. Location of the accelerometer is shown in Fig. 1(a)

figuration. The test board was fixed onto the drop table by four support standoff screws. The schematic of the shock test set up is shown in Fig. 2(a). To apply a shock wave, the drop table was raised and dropped from a calibrated height adjusted on the basis of the peak acceleration half-sine shock pulse with a $1.5 \mathrm{mili}^{-}$ second duration. With an input shock of 200G at the table, the corner of the component experienced a shock level of 300G as shown in Fig. 2(b). The associated strain value at the corner was 1700 microstrain at peak principal strain. An accelerometer was attached to both the table and board as indicated in Fig. 1(a). For both room temperature and $100{ }^{\circ} \mathrm{C}$ elevated temperature tests, the $\mathrm{G}$ level and the strain were maintained at the same level with little difference in principal max strain and max $G$ levels. Each test board run started at 200G shock level until failure. The boards were managed to have the component on topside first with three times in the $+z$ direction and three times in the $-z$ direction after that until the failure occurred. All test boards were monitored in-situ for its electrical continuity during the shock test to detect failures. An increase in resistance of $20 \%$ of the initial resistance value during the test was considered a failure. Tested boards were analyzed by dye-and-pry analysis to observe the failure mode. Optical microscopy and polarized light microscope images were also taken to observe solder joint failures and IMC growth. 

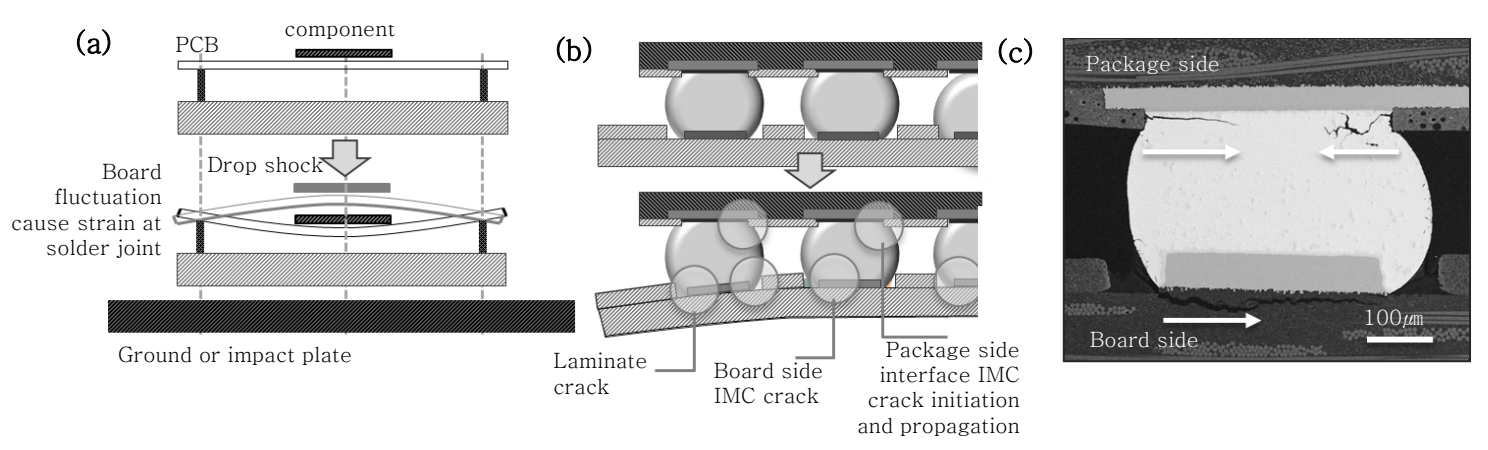

Fig. 3 (a) During shock, the board fluctuation cause strain at the solder joints. (b) Each solder joints have several stress points in each solder interconnection. (c) The example of a corner joint after shock test, which show three different crack propagation/paths: package side near package side interface and board side laminate crack

\section{Results and Discussion}

During dynamic shock, the board flexes to dissipate the shock energy, which entered into the system. The maximum strain appears at the corners of the component, as shown in a simplified illustration in Fig. 3. While the component location on the board is a dominant factor closely related to the strain response induced by the shock wave, the case in this study associated with one component show a less complicated situation with same expected strain levels at each corner of the component. As shown in Fig. 3(b) schematically, there are several, so called, stress points per joint which are high potential fracture sites during shock. It is a continuous competition between each failure mode, which leads ultimately to the full fracture and fail of the joint. The SEM image in Fig. 3(c) reveals the various crack propagation paths in a shock tested corner joint. Three clear crack paths can be identified, two crack paths at the package side from the left and right interface, and a crack propagation at the laminate material under the $\mathrm{Cu}$ pad on the board side. Often, it is the pad design, which define the dominant crack propagation mode, thus this study were conducted on both solder mask defined (SMD) and Non-solder mask defined (NSMD) pad designs at the board side. Addition to the pad design factor, one additional factor is the capability of the solder joint, how much shock energy it can absorb. For example if the solder joint body can absorb more shock induced strain by phase transformation and defect accumulation, the stress points will experience less build up stress/strain, which ultimately leads to a delayed crack initiation and propagation, thus better shock performance.

Fig. 4 shows the initial state SEM micro-
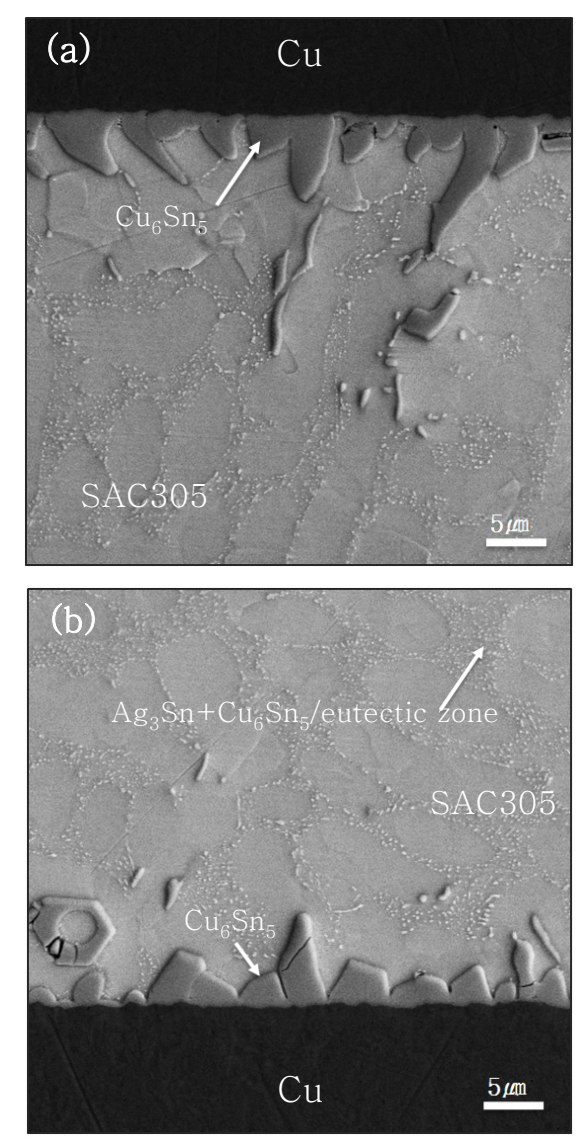

Fig. 4 Initial microstructure SEM image (a) package side interface and (b) board side interface 
structure at the package side and board side interface. Since the surface finish for both side were $\mathrm{Cu}$ OSP, the intermetallic phase at the interface were Cu6Sn5 with scallop shape IMCs. Finely distributed IMC precipitates inside the solder bulk can be seen, which are well know as a mixture of $\mathrm{Ag}_{3} \mathrm{Sn}$ and $\mathrm{Cu}_{6} \mathrm{Sn}_{5}$ IMC precipitates. It has been reported that after isothermal aging, these mixed/eutectic structure are being accumulate and transformed to equiaxed grain structure with larger IMC precipitates in fewer numbers ${ }^{7,8)}$. But in this study, the effects of isothermal aging condition are not covered.

During applying the input table shock of $200 \mathrm{G}$ to the test boards, the continuity of the daisy chain were closely monitored using a data logger and the results are shown in Fig. 5. Three boards were tested for each condition and design variation. As shown in the result plot, the NSMD boards tested at room temperature show a cycle number to failure around 18 to 19 cycles. But immediately degraded to around 5 cycles to failure for the boards tested at $100^{\circ} \mathrm{C}$ elevated temperature, which is a $75 \%$ shock performance degradation.

In case of SMD boards, the cycle to failures occurred in an already low number, around 2 cycles and degraded further to 1 cycle to failure a 50\% degardation. It is clearly shown that the board side pad design implies a large effect

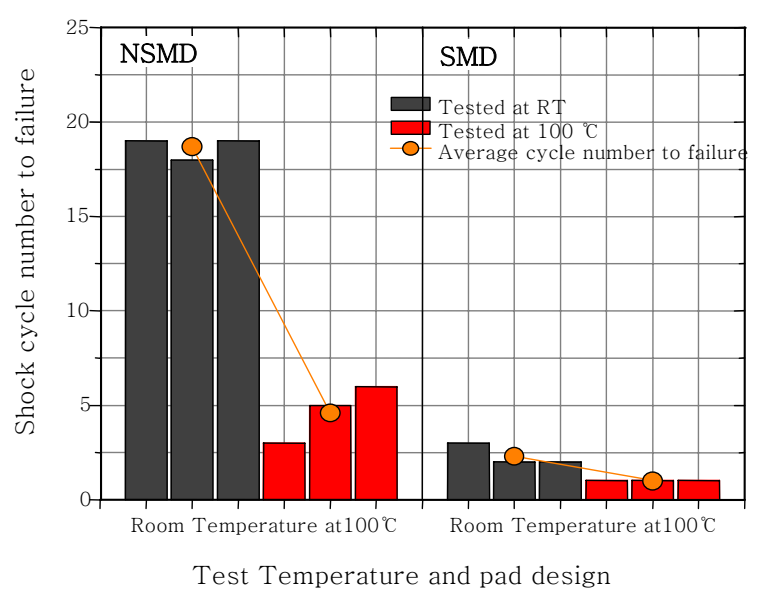

Fig. 5 Shock test results at both room temperature and $100^{\circ} \mathrm{C}$ elevated temperature environment testing condition per board side NSMD and SMD pad design to the shock performance with an identical component configuration. This brings an important design guideline subject to better shock performance for large body size and heavy components.

Fig. 6 shows the dye and pry results on selected components after the shock test to failure. The results show several obvious observations. The first is that the NSMD boards failure modes are dominantly laminate crack propagation. For both room temperature and elevated temperature tested boards, the laminate crack path was the failure mode for NSMD boards. On the other hand the SMD boards show mainly the crack propagation through the board side interface. The detailed crack path will be discussed later in Fig. 8, but it is clear that the failure mode for both room temperature and elevated temperature are at the board side interface. The second observation is that the testing temperature did not made the failure mode shift from one to the other. For both NSMD and SMD, the same failure modes were identified regardless of the testing temperature.

Fig. 7 show the optical images of cross sections from the corner region for NSMD board samples (Fig. 7(a)) and SMD board (Fig. 7(b)). The inset images are the higher magnification polarized optical images from the images above

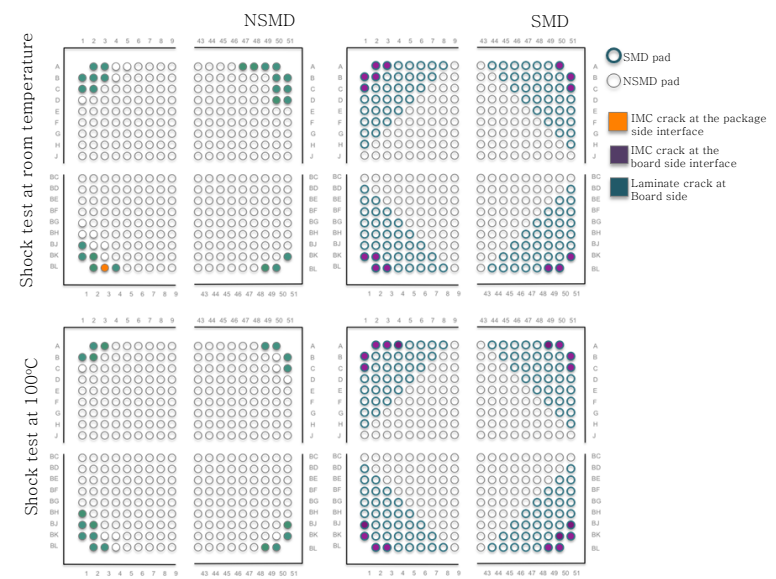

Fig. 6 Dye and pry results at both room temperature and $100^{\circ} \mathrm{C}$ elevated temperature environment testing condition per board side NSMD and SMD pad design 

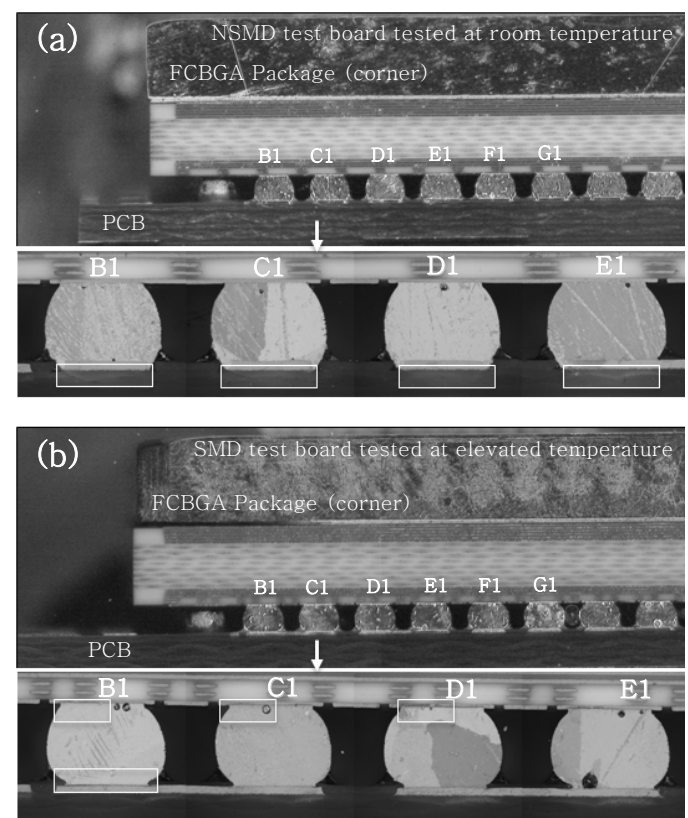

Fig. 7 Cross section optical images after shock test. (a) NSMD board after shock test at room temperature. The inset images are higher magnification Polarized images. (b) SMD board shock tested at $100^{\circ} \mathrm{C}$ elevated temperature. The inset is the polarized images from B1 to E1 joint. B1 joint show twin structure inside the solder bulk region. Crack propagation paths are indicated by white boxes

it. Crack paths are indicated in the box. Aligned with the dye and pry results shown in Fig. 6, the NSMD board solder joints show dominant crack propagation at the laminate region and the SMD a crack propagation at booth package side and board side interface, with a dominant crack path at the board side interface. It is also interesting to identify twin structure development at the B1 joint in Fig. 7(b) inset. A related publication on shock induced twin formation can be found elsewhere ${ }^{9,10)}$.

Fig. 8 is a set of SEM images from a SMD board solder joint after shock test to see crack path/propagation in more detail. The crack initiation can be claimed to be start from the corner of the joint slightly inside the solder bulk area near the interface. It is often a discussion item whether the IMC interface is the crack initiation site for shock induced cracks, but all of the solder joints in this study, which
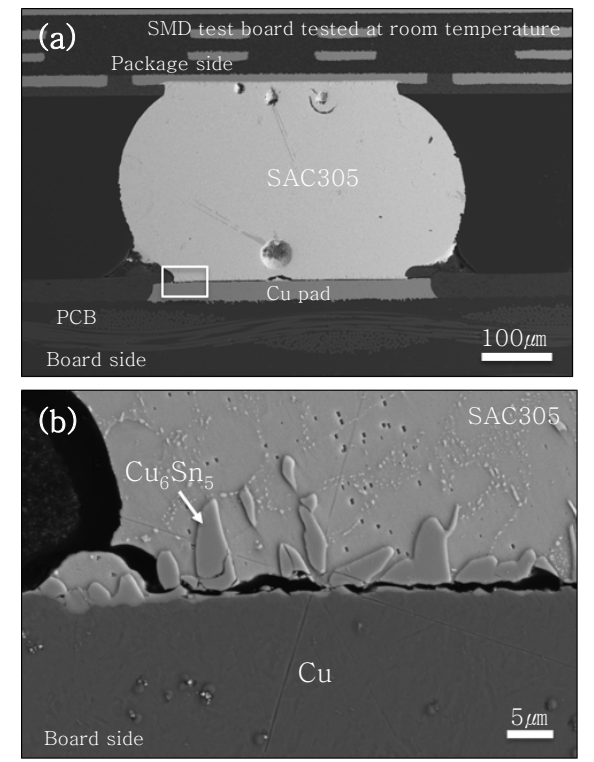

Fig. 8 Cross section SEM images from a selected joint in a SMD board, shock tested at room temperature. (a) overall joint view and (b) higher magnification SEM image of the lower left corner. Region indicated in Fig. 8(a)

have a board side crack propagation, showed the crack initiation from the solder bulk region penetrating into the IMC interface for further propagation. The SEM image in Fig. 8(b) also shows the same representative crack path/propagation, which a crack is initiated from the bulk into the IMC interface and then further propagated through the root of the $\mathrm{Cu}_{6} \mathrm{Sn}_{5}$ IMC scallops.

Comparing to the NSMD boards, this crack propagation in SMD boards are relatively easier than propagation in laminate regions. Addition to that, the crack propagation in IMC has higher possibilities of showing faster electrical discontinuity (by measuring the resistivity during shock test) than laminate crack failure modes. Because the laminate crack mode needs to cut through the $\mathrm{Cu}$ trace to show the full discontinuity, which is expected to be relatively late than the interface solder crack in SMD samples. Overall, the faster crack propagation in boards side interface regions through the IMC root is a fast propagation, which can be the main reason for a shorter life cycle time than NSMD boards. Another important factor is the influence 
of the elevated test temperature. In SMD boards the influence of the elevated test temperature was relatively less than the NSMD, of course the room temperature tested SMD boards showed already a low cycle number to failure at the beginning, but the relatively large degradation for NSMD boards can be explained from two different view points. The first is the laminate material weakening at elevated temperature. The transition glass $(\mathrm{Tg})$ temperature of the laminate material is designed to have a range of 150 to $180^{\circ} \mathrm{C}$, thus an elevated temperature of $100^{\circ} \mathrm{C}$ is too low to cause any major damage to the board epoxy material. But the temperature might have an impact to the interface of the $\mathrm{Cu}$ pad and the epoxy, which can be weakening at an elevated temperature compared to room temperature environment, resulting in an earlier crack initiation at the laminate region. The second view angle is the effect of elevated temperature to the solder joint itself. With a higher temperature, the solder joint bulk material can either be more capable or less capable to absorb the shock induced strain. Also the IMC interfaces can be affected by the elevated temperature test condition. But shown in the shock performance results and failure analysis using dye and pry, the NSMD boards did not show an improvement in shock performance at elevated temperature, thus it seems the capability of shock induced strain absorption was not improved by higher temperature. Also the failure mode was maintained at elevated test temperature, which indicates that the IMC interfaces were not weakened (at least dramatically) at elevated temperature. The results in SMD boards, which failed dominantly at the board side interface is another example that the IMC was not much affected by the elevated temperature. Another possibility explaining these degradations with elevated temperature is the board strain response. The maximum peak $G$ level and principal strain were measured at the same level for both room temperature and $100^{\circ} \mathrm{C}$ elevated temperature test conditions, but the strain cycle frequency and the strain rate reaching the peak strain needs further analysis between those two temperature environment, which will be follow up subject to pursue.

\section{Conclusions}

In this study, the effect of external temperature was observed on large Flip-chip BGA components. The weight and size of the large package produced a high strain region near the corner of the component and thus show full fracture at around 200G level shock input. The shock performance at elevated temperature, showed degradation based on board pad designs. Boards with NSMD pad on the board side show 75\% degradation with elevated test temperature conditions with a dominant laminate crack failure mode. The SMD boards show Board side IMC failure modes for both room temperature and elevated temperature conditions. The overall results revealed several elevated temperature influences including possible laminate (epoxy) weakening at higher temperature leading early laminate crack initiation at elevated temperature condition and less than expected IMC weakening or strengthening at higher temperature to shock induced strain. But since the test in this study was on one shock level (200G), it is too early to conclude the elevated temperature effect, where shock induced strain still can be observed for lower G level shock inputs, which is a topic to look further.

\section{References}

1. J. Glazer, J. Electron. Mater. 23, 8 (1994)

2. W. Xie, T.-K. Lee, K.-C. Liu, and J. Xue, IEEE 60th Electronic Components and Technology Conference (ECTC) (Las Vegas, NV, June 2010)

3. H.G. Song, J.W. Morris Jr., and F. Hua, JOM 56, $30(2002)$

4. D.R. Frear and P.T. Vianco, Metall. Trans. A 25A, 1509 (1994)

5. W.S Hong and C.M.Oh, Journal of KWJS, 31(3), 22(2013)

6. Y.H Ko, M.S Kim, T.S. Kim, J.H Bang, and C.W 
Lee, Journal of KWJS, 31(3), 4 (2013)

7. Tae-Kyu Lee, Hongtao Ma, Kuo-Chuan Liu and Jie Xue, J. Electron. Mater. 39, 2564 (2010)

8. T.-K. Lee, W. Xie, B. Zhou, T. Bieler, and K.-C. Liu, J. Electron. Mater. 40 (9), 1967 (2009)

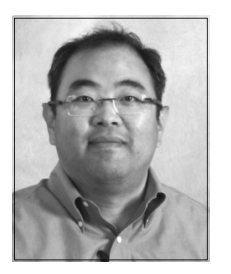

Tae-Kyu Lee is a Reliability Engineer in Cisco Component quality and technology (CQT) group, Packaging and Interconnect Technology Team located in San Jose, CA. Since he joined Cisco in 2006, he is working on board level interconnect reliability, next generation packaging technology and solder material characterizations. Tae-Kyu received his Ph.D. degree in Materials Science and engineering from University of California, Berkeley on studies related to high temperature Superconducting Quantum Interference Device (SQUID) non-destructive evaluation and was a Postdoctoral fellow in Lawrence Berkeley National Laboratory before joining Cisco. He is actively involved in several industry consortia and as a lead organizer for the Lead free solder technology workshop, for the last five years, and serving as a Vice Chair in the Electronic Packaging and Interconnect Technology Committee in TMS. He is also an associate editor in Journal of Electronic Materials.
9. E. El-Danaf, S.R Kalidindi, R.D. Doherty, Metal. and Mat. Trans. 30A, 1223 (1999)

10. T.-K. Lee, B. Zhou, T. Bieler, C.Tseng, and J. Duh, J. Electron. Mater. 42 (2), 215 (2013) 